\title{
El concepto social de climaterio en población rural y urbana
}

\author{
Ana $\mathrm{M}^{\mathrm{a}}$ Carrobles, Minerva Velasco y Sagrario Gómez-Cantarino \\ Universidad de Castilla-La Mancha (España)
}

El climaterio es una fase de transición compleja en la que intervienen factores biológicos, psicológicos, sociales y culturales. El fenómeno del climaterio es algo natural y universal para todas las mujeres. Esta etapa está influenciada por el entorno socio-cultural en el que la mujer viva. A través de un estudio de investigación cualitativo entre mujeres residentes en la provincia de Toledo. La selección de mujeres se realizó mediante un muestreo no probabilístico de conveniencia. El procedimiento grupos de discusión con las mujeres, de diferente condición social, y de situaciones personales diferentes. Las mujeres de ámbito urbano en su mayoría ven el climaterio como algo patológico, muchas de ellas necesitan ayuda para afrontar este momento, sin embargo, las de zonas rurales ven el climaterio como algo natural, otra etapa más de sus vidas en parte porque las redes de apoyo familiares aportan vivencias y experiencias que comparten con sus familias. Las de zonas urbanas llegan después el climaterio, las multíparas desarrollan más tarde la menopausia. Las amas de casa llegan antes al climaterio, que las que trabajan fuera de casa. Dependiendo de la actitud frente al climaterio, van a poder tener unas experiencias u otras, A través del conocimiento del concepto de climaterio, mejora en el futuro la calidad asistencial.

Palabras clave: Envejecimiento, climaterio, menopausia, ansiedad, proceso natural.

The social concept of climacteric in rural and urban population. The menopause is a phase of complex transition that involves biological, psychological, social, and cultural factors. The phenomenon of the menopause is something natural and universal for all women. This stage is influenced by the socio-cultural environment in which the woman to live. Through a qualitative research study among women who live in the province of Toledo. The selection of women was performed by sampling non-probability convenience. The procedure of discussion groups with women, of different social status, and various personal situations. Urban women are mostly the menopause as something pathological, many of them need help to deal with this moment, but those in rural areas see the menopause as a natural, another stage more of their lives in part because family support networks provide experiences and experiences that they share with their families. The urban areas arrive after the climacteric, multiparous women develop after menopause. Housewives become before the menopause, those working outside the home. Depending on the attitude towards the climacteric, will be able to have experiences or others, through the knowledge of the concept of menopause, improves in the future the quality of care.

Keywords: Aging, climacteric, menopause, anxiety, natural process.

Correspondencia: Ana $M^{a}$ Carrobles García. Facultad de Terapia Ocupacional, Logopedia y Enfermería. Universidad de Castilla-La Mancha. C.P. 45006. Toledo (España). E-mail: AnaMaria.Carrobles@uclm.es 
El climaterio, comprende la tercera parte de la vida de la mujer, llena de vivencias y cambios, tanto a nivel físico como psíquico, acaba el periodo reproductivo, llega la menopausia y, además, la mujer debe asociar nuevos cambios y roles en su vida. $\mathrm{Y}$ cada una de ellas tiene una forma de enfrentarse a esta etapa, sus emociones, actitudes dependiendo de su manera de ser y también del entorno social que les rodea. Mientras que los aspectos físicos que viven son muy similares: sofocos, irritabilidad, insomnio, etc., muchas veces debido a la bajada de estrógenos que ocurren en esta etapa. Las capacidades y su afrontamiento psicológico a la menopausia o perimenopausia varían (Perandones y Larroy, 2011).

La Federación Internacional de Ginecología y Obstétrica (FIGO) lo define como "la fase del proceso de envejecimiento durante la cual, la mujer pasa de su etapa reproductiva a la que ya no lo es" (Munro y Critchley, 2011).

Según la Organización Mundial de la Salud (OMS) el Síndrome Climatérico es el conjunto de síntomas y signos que aparecen en el período de transición que precede y acompaña a la pérdida de la capacidad reproductiva, hasta llegar a una involución significativa de la función ovárica. (OMS, 1981).

Es una de las épocas más importantes en la vida de la mujer. Para algunas es un reto por las dificultades físicas y emocionales, para otras es un momento de plenitud personal, y para otras muchas una combinación de lo anterior que requiere un esfuerzo adicional y el apoyo de su entorno familiar, laboral y social (García-Viniegras, Regina, y Porta, 2003).

Muchas investigaciones utilizan el estado de salud autopercibido para acercarse al concepto de salud de las personas. Esta percepción subjetiva se establece al comparar la salud de uno, con el ideal (Molina, Vílchez, Velásquez, y Fernández, 1999). Varios autores sostienen que en España el porcentaje de mal estado de salud subjetivo es mayor en las mujeres que en hombres desde las últimas décadas, y que los porcentajes son más elevados en personas sin estudios, respecto a personas con estudios de segundo o tercer grado (Lezaun, 2006). A esto se añade que la buena salud subjetiva disminuye con la edad. Así, desde el punto de vista epidemiológico, será una de las variables a utilizar a la hora de investigar la concepción de la salud durante esta etapa concreta de la vida de las mujeres. El conocimiento sobre su estado de salud percibido, así como las creencias, valores y significados que acompañan al climaterio, es esencial en el cuidado de su salud y, por consiguiente, en la valoración sanitaria integral de atención primaria como de la atención especializada.

Hasta hace sólo algunas décadas, las mujeres veían la menopausia como un hecho real y biológico que reconocían y manejaban gracias a un sistema de autocuidados construido culturalmente. En la actualidad, nuestra sociedad ha elaborado un discurso según el cual la menopausia es un proceso patológico, lleno de riesgos para las mujeres y, por lo tanto, susceptible de tratamiento (Agramonte, Navarro, y Domínguez, 2005). 
Gutiérrez (2003) señala que "escuchamos a menudo bromas hirientes y discriminantes o reproches que suelen hacerse sobre los cambios de humor, irritabilidad y depresión de las mujeres, para justificar conductas incomprendidas para la sociedad”. Esta manera de pensar que manifiestan las mujeres, es por la visión de pérdida que sufren al terminar la etapa reproductiva, ya que viven la menopausia como una etapa de pérdida de la capacidad reproductiva donde es duro encajar que llega la vejez y ya no sirves para traer al mundo más hijos.

Existen grandes diferencias geográficas en la prevalencia de la sintomatología y algunas diferencias en edad de aparición de la menopausia. Tanto en Asia como en América Latina, las mujeres de nivel socioeconómico más pobre, inician mucho antes de la menopausia, las diferencias regionales en la edad de la menopausia y climaterio son importantes. Hay que reconocer y sentar las bases del impacto en la salud de la mujer en las diferentes regiones del mundo (Regidor et al., 2006).

Los resultados de programas actuales de intervención en mujeres climatéricas, evidencian una mejora en su calidad de vida, desarrollan una sensación de tranquilidad que tiene que ver con la madurez, autoestima, y mayor confianza en ellas mismas. Esta es la mejor etapa para adquirir nuevos saberes y la necesidad de apoyo y educación para la salud en estos momentos de cambios (Álvarez, Martín, Emma, y Bordones, 2008).

\section{Objetivo}

El presente estudio cualitativo pretende explorar a la mujer, en el climaterio, tanto en las zonas rurales como urbanas, y analizar como el entorno socio-cultural influye en sus vivencias en esta etapa.

\section{MÉTODO}

Estudio de investigación cualitativa descriptiva de carácter fenomenológico, el cual tiene su origen en la corriente filosófica de Husserl. La fenomenología tiene su objetivo en pensar en cómo son las experiencias de vida de las personas. El investigador fenomenologista se pregunta ¿Cuál es la esencia de este fenómeno según lo experimentan estas personas? Por tanto, el objetivo de la investigación fenomenológica consiste en describir plenamente la experiencia vivida y las percepciones a las que dan lugar, con el fin de obtener un conocimiento holístico y profundo de la realidad abordada. Es el mejor método para que la mujer exprese sus vivencias y experiencias, ya que eso nos enriquece y llegamos a conocer la realidad de las mujeres climatéricas.

\section{Participantes}

Mujeres de 45 a 65 años pertenecientes al Área Sanitaria 1 de Toledo. De ámbito rural y urbano, estas mujeres pertenecen a un variado espectro socioeconómico. 
La selección de mujeres se realizó mediante un muestreo no probabilístico de conveniencia, al ser un método fácil y eficiente.

\section{Instrumento}

Grupos de discusión. Los grupos de discusión son un método convencional de recogida de datos cualitativos (Salamanca, 2013). Para el posterior análisis de los datos, se grabó con una grabadora, con la autorización de las mujeres, toda la información. Y, después de transcribirla literalmente, fue analizada.

\section{Procedimiento}

Mediante grupos de discusión, tanto en el ámbito urbano como el rural, con 9 y 12 mujeres. Formamos grupos heterogéneos en cuanto a edad, nivel educativo, diferentes creencias y religiones, con diferentes sentimientos, actitudes y vivencias para conocer la realidad de un colectivo de mujeres que demandan nuestros cuidados de salud.

\section{Análisis de datos}

Al organizar la información recogida, encontramos respuesta al objetivo del estudio. La información se analizó simultáneamente a su recogida. Con el fin de identificar patrones y conceptos importantes, qué cosas nos faltaban por saber y qué no encaja con el análisis realizado. Conseguimos la saturación de la muestra, ya que después de una hora no nos aportaban nada nuevo. En los estudios cualitativos es el método inductivo el que se utilizó, llegando a la siguiente realidad: según las mujeres entrevistadas, esto es lo que pudimos deducir de sus palabras.

Gráfico 1. Síndrome climatérico

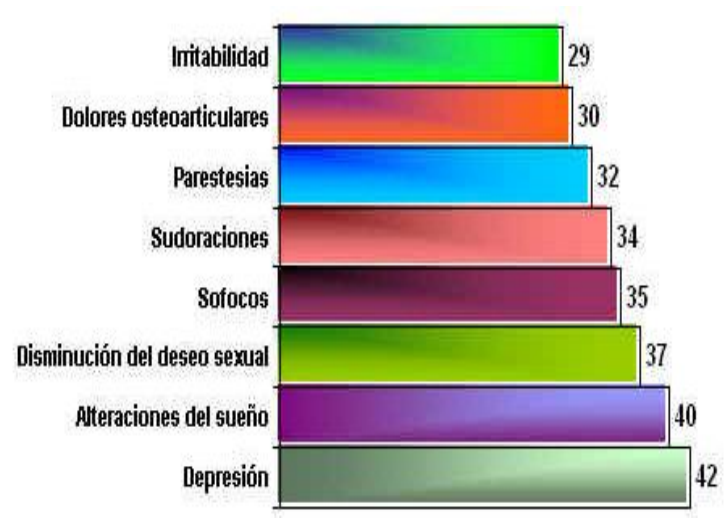




\section{RESULTADOS}

El 29\% de las mujeres reconocieron estar irritables en el trato hacia los demás El 30\% de ellas reconocieron tener dolores osteoarticulares. El 32\% nos reconocieron tener parestesias. Un mayor número, $34 \%$ de ellas, reconocieron padecer sudoración frente a un 35\% que lo definió como sofocos, con lo cual más del $69 \%$ reconoció tener sofocos o sudoración anormal en esta etapa de sus vidas. En torno a un $37 \%$ de las mujeres del grupo de discusión reconoce la disminución del deseo sexual, se definen como mujeres secas sin necesidad de tener relaciones con sus parejas. Un $40 \%$ del grupo afirma tener alteraciones del sueño y un $42 \%$ sufre depresión y la necesitad de ayuda farmacológica para poder seguir su vida. Con lo cual, vemos que el climaterio es una etapa en la que la totalidad de mujeres que han sido estudiadas muestran signos y síntomas característicos de esta etapa, pero que cada una de ellas lo experimenta y lo vive de un modo diferente.

\section{DISCUSIÓN Y CONCLUSIONES}

En el climaterio cada mujer vive de forma diferente la aparición, intensidad y duración de los signos y síntomas, de la etapa climatérica. Esto está influido por los factores socioculturales que marcan a cada mujer. Pero también debemos valorar que hay mujeres que no manifiestan estas vivencias. Así, tras realizar el estudio, podemos decir que dependiendo del nivel sociocultural de la mujer, si vive en una zona rural o urbana, si tiene pareja o no, si tienen nietos o familiares a los que deben atender a la vez que trabajar dentro o fuera de casa, vive de una forma u otra esta etapa. Este estrés que manifiesta muchas veces, crea problemas sociales, conyugales y personales. La mayoría de las mujeres manifestaron ansiedad, irritabilidad, problemas en el ámbito familiar y sexual (Lugones y Navarro, 2006). Vemos que hay diferentes formas de vivir esta etapa.

España es uno de los países que envejece a más velocidad. Dicho envejecimiento se produce principalmente debido a tres tendencias: una de ellas está producida por la fecundidad, por otra parte, España presenta una de las esperanzas de vida tras los 65 años más altas de Europa y, por último, ha sido uno de los países a nivel Europeo que más inmigración ha recibido (López, 2011). Por esto es necesario que conozcamos en profundidad el climaterio, para poder ser capaces de atender desde la atención primaria de salud a todas las mujeres que demandan asistencia sanitaria en la edad del climaterio. Hay que pensar que con la calidad de vida que tenemos en el país, la mujer vive en el climaterio una tercera parte de su vida, lo que hace necesario tener más habilidades y destrezas para tratar a las mayores demandantes de salud.

Hasta hace unas decadas, el climaterio se veía como algo normal en la vida de la mujer y esto no ha cambiado en las zonas rurales gracias al sistema familiar, ya que las primas, hermanas, abuelas, madres o vecinas enseñaban o relataban sus experiencias en la 
puerta de sus casas con total normalidad. El sistema de autocuidados construido culturalmente funcionaba muy bien. Pero esta realidad en las zonas urbanas, donde la mujer trabaja fuera de casa, donde no se relacionan de igual manera que en las zonas rurales, ha evolucionado y tiene una visión de etapa patológica, donde lo mejor que puede hacer la mujer es buscar ayuda para afrontar esta etapa en el sistema sanitario. La gran ventaja de esta visión la tienen las empresas farmacéuticas que venden todo tipo de fármacos y remedios para paliar los signos y síntomas de la menopausia y el climaterio. Pero no podemos dejar que esto siga ocurriendo en el siglo XXI, y más sabiendo la poca utilidad de los fármacos muchas veces funcionan como efecto placebo. Es la propia mujer la que debe tomar las riendas de su vida, vivir este momento como un gran reto y una etapa de total desarrollo, tanto intelectual como físico. Y las enfermeras tenemos mucho que hacer en este tema, ya que debemos empoderar a la mujer y hacerla responsable de su autocuidado y devolverle la visión de etapa normal, olvidando la visión de patología. La crisis nos está dando una gran oportunidad. No es solo una crisis económica, como en principios pensamos, es algo más fuerte, es un cambio de visión, tanto a nivel social como económico. Debemos retomar antiguos valores que habían cambiado y recuperar la esencia de la mujer. Los grupos de trabajo que se crean en los centros de salud y en las consultas de enfermería funcionan muy bien, las mujeres se unen porque comparten experiencias, vivencias y creencias. Las enfermeras somos como árbitros en estos grupos, no somos las que más sabemos, porque muchas veces las propias mujeres tienen un conocimiento más profundo sobre su cuerpo y sus experiencias que nosostras por ser personal sanitario. Pero con esta manera de trabajar, donde son ellas mismas las que cogen las riendas de sus vidas y deciden desde sus propias motivaciones resurgir como mujeres más sabias, más preparadas para vivir una etapa bellísima de sus vidas.

Pensemos que el tema es un problema de salud pública mundial ya que el $80 \%$ de las mujeres experimentan síntomas físicos o psicológicos, cuando alcanzan la edad de la menopausia y entran en el climaterio. Cuando la calidad de vida que tenemos y el bienestar de las mujeres de mediana edad debería ser cada día mejor. Pongamos desde nuestras consultas de enfermería, ese granito de arena que haga que la realidad cambie y todas las mujeres vivan este momento plenamente, desde nuestras consultas debemos capacitarlas para que esto, sea una realidad.

\section{REFERENCIAS}

Agramonte, A., Navarro, D., y Domínguez, E. (2005). Menopausia, Sexualidad Mujer de edad mediana con Diabetes Mellitus. Revista Ecuatoriana de Ginecología y Obstetricia, 11(2), 25-29.

Álvarez, R., Martín, E., y Bordones de Álvarez, M. (2008). Conocimiento y actitudes sobre el climaterio en mujeres entre 40 y 50 años. Revista Obstétrico Ginecológica Venezuela, 68(1), 32-40. 
García-Viniegras, V., Regina, C., y Porta, M. (2003). Salud. Climaterio y bienestar psicológico. Revista Cubana Obstétrica Ginecológica, 29(3), 87-96.

Gutiérrez, E. (2003). Las mujeres, el climaterio y sus percepciones. Revista Obstetricia y Ginecología Colombia, 9(4), 24-37.

Lezaun, J.Y. (2006). Análisis de la calidad de vida relacionada con la salud en la vejez desde una perspectiva multidimensional (Vol. 11002). Instituto de Mayores y Servicios sociales.

Lugones, M., y Navarro, D. (2006). Síndrome climatérico y algunos factores socioculturales relacionados con esta etapa. Revista Cubana de Obstetricia y Ginecología, 32(1).

Molina, R., Velásquez, N., y Fernández, G.E. (1999); Diagnóstico y seguimiento clínico durante el climaterio y la menopausia. En J. Terán Dávila y F. Febres Balestrini. Medicina del Climaterio y la Menopausia. Caracas. Ediciones Ateproca; 15-34.

Munro, M.G., Critchley, H.O., y el Grupo, I.S.F. (2011). La clasificación FiGo de causas de sangrado uterino anormal en los años reproductivos. Revista del Climaterio, 15(85), 9-17.

Organización Mundial de la Salud OMS (1981); "Investigación sobre la menopausia". Serie de informes técnicos $n^{\circ} 670$. Ginebra: OMS.

Perandones, M., y Larroy, C. (2011) Intervención grupal para la mejora de la respuesta sexual y la calidad de vida de mujeres en climaterio: un estudio piloto. Revista Psicopatología y Psicología Clínica; 6(3), 159-168.

Regidor, E., Martínez, D., Astasio, P., Ortega, P., Calle, M.E., y Domínguez, D. (2006). Evolución de las desigualdades socioeconómicas y las desigualdades en la percepción de la salud en España. Gaceta Sanitaria, 20(3), 1-5.

Salamanca, A.B. (2013) El aeiou de la investigación en Enfermería. Rivadeneyra. Fuden, 269-272.

Vicente, J.M. (2011). Libro blanco sobre envejecimiento activo. $1^{a}$ ed. Madrid. Ministerio de Sanidad. Política Social e Igualdad. Secretaría General de Política Social y Consumo. Instituto de Mayores y Servicios Sociales (IMSERSO). 